\title{
Il Museo della lingua italiana: una svolta nella politica linguistica nazionale?
}

\author{
Nicoletta Maraschio
}

PUBBLICATO: 30 AGOSTO 2020

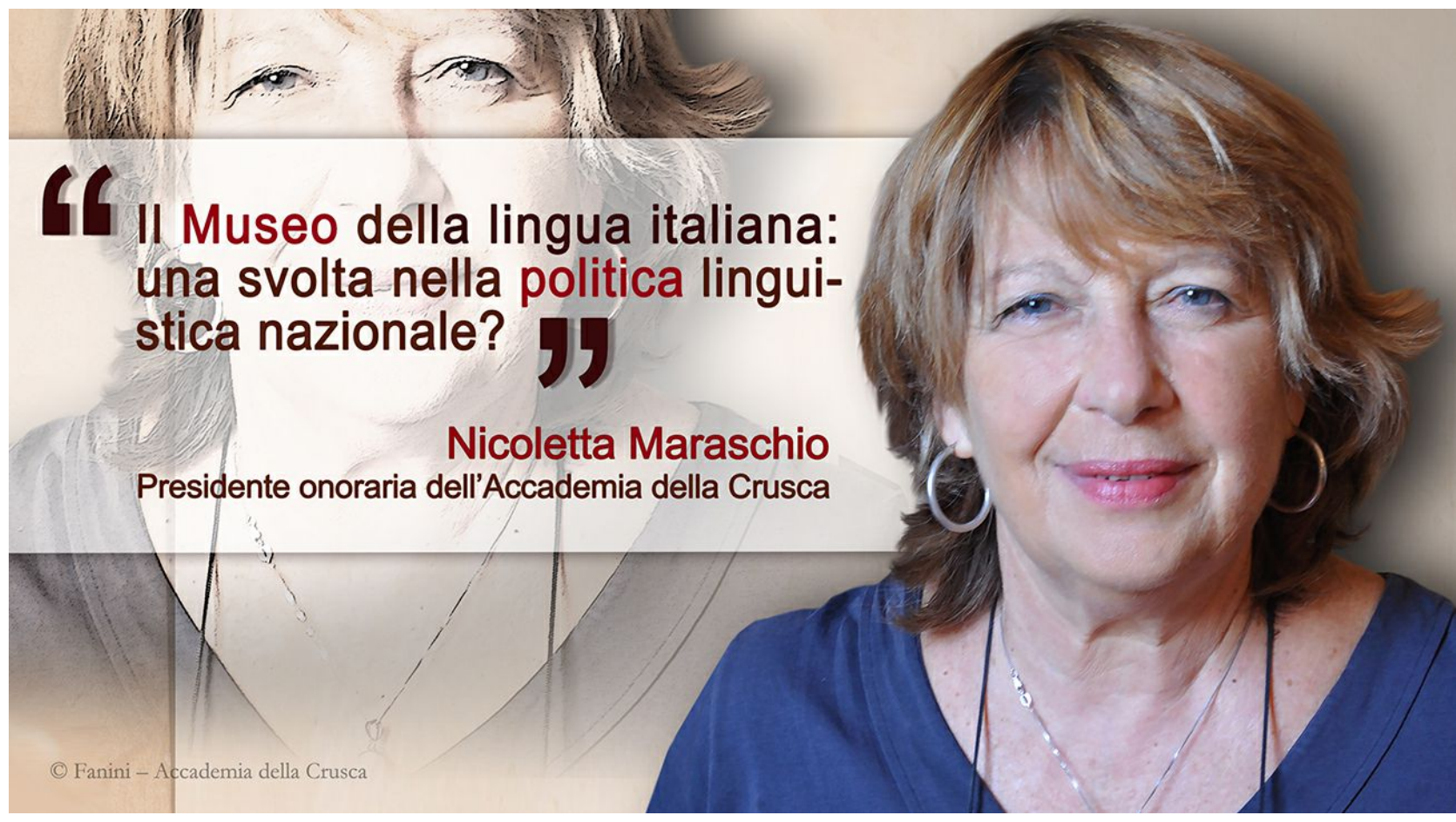

0 i può fare un museo di un bene immateriale in perenne movimento come una lingua? Non c'è il rischio di fissarlo in un percorso espositivo necessariamente riduttivo e semplificante? Molti pensano che anzi un museo possa essere uno strumento straordinario di conoscenza, tutela e valorizzazione della lingua. Il tema è a mio avviso affascinante e merita di essere dibattuto. Un argomento a favore del museo della lingua è che nel mondo ne esistono già molti. In Italia se ne parla da tempo, ma il "sogno" si è concretizzato nella notte delle stelle cadenti di San Lorenzo (l'immagine suggestiva si deve a Giuseppe Antonelli, autore del fortunato libro Il Museo della lingua italiana, Mondadori 2018).

Dopo tante riflessioni, iniziative e pubblicazioni, dopo i lavori propositivi di un gruppo qualificato di studiosi (coordinato da Luca Serianni), finalmente il progetto del primo Museo della lingua italiana prende corpo. La notte di San Lorenzo ha portato due grandi novità: il finanziamento e la sede, grazie a un accordo tra il Ministro Dario Franceschini, il Sindaco di Firenze Dario Nardella e l'Assessore alla cultura Tommaso Sacchi. Si passa dall'astrattezza di progetti sulla carta alla concretezza della loro fattibilità in un luogo preciso. Anche l'Italia avrà dunque il suo Museo della lingua (Per altri musei nel mondo si veda Lucilla Pizzoli, I musei nel mondo dedicati alle lingue, Istituto della Enciclopedia Italiana 2018 e Margaret Sönmez, Maia Wellington Gahtan, Nadia Cannata (a cura di), Museums of Language and the Display of Intangible Cultural Heritage, Routledge 2020).

In precedenza, si contano diverse iniziative per "far vedere" la nostra lingua e per ripercorrerne la storia attraverso immagini e oggetti: dalla mostra agli Uffizi Dove il si suona (2003) a quella dedicata all'Homo sapiens (Roma, Palazzo delle Esposizioni 20II-20I2, con ampio spazio per la Storia linguistica 
italiana), fino al percorso espositivo alla Sapienza di Roma, curato da N. Cannata, M. Gahtan e dai loro allievi (Dalla mostra al museo: Eurotales, il museo interattivo delle lingue d'Europa, 2org). Il Vittoriano, inoltre, è stato sede di almeno due esposizioni importanti: la prima (20II) curata da Francesco Sabatini e inaugurata dal Presidente Ciampi (L'unità della lingua e l'unità della Nazione) e la seconda, più recente (2019), Lessico italiano. Volti e storie del nostro Paese, a cura di Edith Gabrielli. E poi ci sono le pubblicazioni: i volumi della Storia della lingua italiana per immagini (diretti da Luca Serianni, zoro segg.) e il portale VIVIT, nel sito dell'Accademia della Crusca (20I3), un vero e proprio museo virtuale e interattivo, realizzato grazie al contributo di un nutrito gruppo di studiosi con competenze diverse.

Il nuovo Museo nascerà a Firenze nel complesso monumentale di Santa Maria Novella, davanti alla Stazione ferroviaria. I luoghi hanno, lo sappiamo, un forte potere simbolico ed evocativo. La prima pietra della Basilica domenicana pare sia stata posta nel I279, esattamente ventisette anni dopo la coniazione del fiorino d'oro (I252), emblema della potenza economica della città medievale, alla quale è strettamente legato lo sviluppo di una civiltà della scrittura volgare unica in Europa. Proprio quella civiltà che avrebbe favorito nel Trecento la creazione dei tre capolavori letterari che sono alla base dell'intera storia linguistica italiana (Commedia, Canzoniere, Decameron). La facciata della Basilica si deve a Leon Battista Alberti, autore della prima grammatica del toscano parlato. La Stazione ferroviaria è il capolavoro razionalista di un altro grande architetto, Giovanni Michelucci, costruita negli anni Trenta del Novecento, in un periodo di grande fervore culturale di Firenze, quando Montale dirigeva il Gabinetto Vieusseux e scrittori e poeti illustri si incontravano alle Giubbe Rosse. Si tratta di una delle stazioni ferroviarie più grandi d'Italia, frequentata da milioni di viaggiatori italiani e stranieri. Medioevo, Rinascimento e Contemporaneità sono dentro e fuori il nuovo Museo, in un polo destinato a diventare attrattivo per un pubblico ampio; in primo luogo per le scuole e per tutti gli "amatori" della lingua italiana, ma anche per quei turisti e visitatori che siano desiderosi di uscire dagli itinerari più consueti e affollati della città d'arte.

Conviene, tuttavia, sgomberare subito il campo da ogni equivoco. Firenze è stata fondamentale nella storia della nostra lingua (basti pensare, appunto, a Dante, Petrarca e Boccaccio), ma il Museo avrà certamente carattere nazionale e internazionale. Litaliano nel mondo continua a essere amato e studiato. Eppure la nostra lingua ha bisogno di essere meglio conosciuta e valorizzata ovunque, in particolare proprio in Italia, e i giovani sono chiamati a diventare protagonisti di una nuova fase della nostra storia linguistica.

Da questo punto di vista il Museo rappresenta un atto straordinario di politica linguistica, tanto più apprezzabile alla luce della poca attenzione finora riservata dallo Stato alla nostra lingua: bene culturale fondamentale che, al pari delle altre lingue di cultura, merita di essere sostenuto, tutelato e valorizzato in modo permanente e organico. Soprattutto ora, di fronte alla globalizzazione e in coerenza con una politica linguistica europea che, almeno astrattamente, sostiene il valore del multilinguismo/multiculturalismo.

In diversi interventi abbiamo letto di un museo multimediale e interattivo, con laboratori didattici d'avanguardia. Certo. Sappiamo che ogni lingua è un bene immateriale in continuo movimento, un bene che tuttavia ha lasciato nel tempo e nello spazio tracce materiali consistenti. Le tracce dell'italiano le troviamo sparse nell'intera Penisola e al di fuori dei nostri confini. Dalla toponomastica al visibile parlare inciso su monumenti, chiese e palazzi; dai manoscritti medievali, conservati in archivi e biblioteche, alle registrazioni del parlato pubblico novecentesco e contemporaneo (quello del teatro, del cinema, della radio, della televisione), fino all'italiano cantato dell'opera lirica che ci ha resi famosi nel mondo. Per non parlare dei tanti italianismi che sono una componente essenziale del comune 
patrimonio linguistico europeo. Per secoli l'Italia, infatti, è stata ponte tra le lingue classiche (greco e latino), quelle del Mediterraneo (soprattutto arabo, insieme alla Spagna) e le altre lingue d'Europa. Si studierà il modo di collegare queste tracce, creando una rete di applicazioni diffuse. Il Museo guiderà ogni visitatore a ritracciare i segni di vicende linguistiche secolari, a riconoscerli e osservarli. Da Firenze al mondo, dal mondo a Firenze, perché nel Museo si esporranno naturalmente anche molti beni materiali significativi: manoscritti, oggetti, libri, quadri.

Si è insistito anche su un museo aperto a tutte le varietà: dalla lingua della letteratura e della scienza a quella del diritto e della politica, dalla lingua dell'arte e della musica a quella della predicazione, fino a quella della cucina. Certo. Tuttavia credo sia essenziale non perdere di vista un obiettivo prioritario: valorizzare le due caratteristiche fondamentali della nostra storia, ossia il multilinguismo e la continuità. Per quanto riguarda la coesistenza di più lingue, è stato notato qualche anno fa da Tullio De Mauro che l'indice di diversità linguistica in Italia è oggi particolarmente elevato: pari allo o,59 e quindi superiore a quello di Svizzera $(0,45)$, Austria e Spagna $(0,44)$, doppio rispetto a quelli di Turchia (0,28), Russia e Francia (0,27) e triplo rispetto a quello della Germania (o,I9). Un multilinguismo diffuso e persistente (in qualche modo parallelo alla biodiversità tipica del nostro Paese), costituito dai molti volgari medievali, e poi da italiano, dialetti, lingue minoritarie, lingue straniere e più recentemente da lingue immigrate, che evolvono in un rapporto fruttuoso di interscambio, sovrapposizioni e contaminazioni.

Quanto alla continuità, essa è è specifica dell'italiano, caratterizzato da un'estensione cronologica, dal Medioevo alla Contemporaneità, estranea alle altre lingue europee, le quali conoscono una frattura netta tra la fase antica e quella moderna. Sappiamo che una illustre tradizione linguistica (letteraria e scientifica), da Dante ad Ariosto, da Galileo a Goldoni, da Manzoni a Montale ci ha portato nel mondo; per non parlare delle parole dell'arte: Alberti, Piero della Francesca, Leonardo, Vasari... fino ai futuristi. Ma l'italiano è stato ed è anche lingua popolare, scritta e parlata dalle generazioni di donne e uomini che hanno abitato la Penisola e che l'hanno usata, talvolta con fatica, soprattutto per comunicare con chi stava altrove. Le loro lettere, e in genere le loro scritture private, sono una testimonianza preziosa dell'esistenza di una lingua comune italiana che era diffusa ben prima dell'Unità d'Italia. Studi recenti hanno aperto e allargato questa interessante prospettiva storiografica e il Museo dovrà darne opportuna testimonianza.

Entrambi questi elementi, multilinguismo e continuità, ci distinguono nel mondo, rappresentano una ricchezza della nostra storia e insieme una grande potenzialità che ci proietta nel futuro, al di là delle troppo pessimistiche previsioni della morte prossima della nostra lingua. Il Museo, che naturalmente mostrerà anche le novità linguistiche del presente portate dalla rete (e da una connessione comunicativa permanente ed estesa come non mai), sarà il luogo ideale per far conoscere a un pubblico largo, e in particolare ai giovani, un patrimonio in gran parte sconosciuto, per far crescere la consapevolezza del suo enorme valore e quindi per suscitarne o rafforzarne l'amore. In questo particolare momento, in cui è urgente avviare imprese culturali innovative, la creazione del primo Museo della lingua italiana, a mio avviso, ha un grande significato e un valore incontestabile. Il cantiere è aperto. Occorrerà naturalmente pensare a una forma giuridica adeguata e soprattutto a un disegno architettonico, ad allestimenti e piu in generale a una filosofia espositiva che non solo siano in armonia con lo straordinario contesto circostante, ma anche in grado di stimolare, in grandi e piccini, tutte le curiosità e le emozioni che la nostra più che millenaria storia linguistica merita.

[“"una versione più breve di questo intervento è stata pubblicata su "Il Fatto Quotidiano" (22 agosto 2020)] 


\section{Cita come:}

Nicoletta Maraschio, I/ Museo della lingua italiana: una svolta nella politica linguistica nazionale?, "Italiano digitale", 2020, XIV, 2020/3 (luglio-settembre)

DOI: $10.35948 / 2532-9006 / 2020.4377$

Copyright 2020 Accademia della Crusca

Pubblicato con licenza creative commons CC BY-NC-ND 